\title{
A PHD in immunosuppression: oxygen-sensing pathways regulate immunosuppressive Tregs
}

\author{
Weiping Zou ${ }^{1,2,3,4}$ and Yatrik M. Shah ${ }^{4,5,6}$ \\ 'Department of Surgery, ${ }^{2}$ Department of Pathology, ${ }^{3}$ Center of Excellence for Cancer Immunology and Immunotherapy, ${ }^{4}$ Rogel Cancer Center, ${ }^{5}$ Department of Molecular and Integrative Physiology, and \\ ${ }^{6}$ Department of Internal Medicine, University of Michigan, Ann Arbor, Michigan, USA.
}

\begin{abstract}
The oxygen-sensing prolyl hydroxylase domain (PHD) enzymes are key to maintaining tissue homeostasis during hypoxia via their regulation of the expression and activity of HIF, the master transcription factor for the hypoxic response. In this issue of the JCI, Yamamoto, Hester, and colleagues show that temporal and reversible inhibition of PHD2 in vivo leads to systemic autoimmune disorder. The work demonstrates that a reduction of PHD2 leads to impairment of immunosuppressive Treg function via a HIF2 $\alpha$-dependent mechanism, without altering Foxp3 expression. This study indicates that a PHD2/HIF2 $\alpha$ axis is critical for maintaining proper Treg function.
\end{abstract}

\section{Oxygen-sensing pathways}

The cellular response to hypoxia is regulated by the heterodimeric transcription factor HIF. HIF consists of a constitutively expressed HIF1 $\beta$ subunit, which is encoded by the aryl hydrocarbon receptor nuclear translocator (ARNT) gene, and an oxygen-sensitive $H I F \alpha$ subunit. The two best-characterized $\alpha$ subunits are HIF $1 \alpha$ and HIF $2 \alpha$ (1). HIF is regulated by oxygen via the action of prolyl hydroxylase domain (PHD) enzymes, which are ironand $\alpha$-ketoglutarate-dependent dioxygenases that utilize molecular $\mathrm{O}_{2}$ for hydroxylation reactions. Under adequate cellular oxygen, iron, and $\alpha$-ketoglutarate levels, PHDs hydroxylate $\mathrm{HIF} \alpha$ on conserved proline residues. Hydroxylation leads to the binding of von Hippel-Lindau (VHL) protein and recruitment of an E3 ubiquitin ligase complex, resulting in rapid degradation of HIF (2). During hypoxia, HIF $\alpha$ is stabilized, binds to ARNT, and regulates target genes that control angiogenesis, cellular metabolism, iron handling, survival, proliferation, and migration. The three
PHD isoforms PHD1, PHD2, and PHD3 (also termed EGLN2, EGLN1, and EGLN3, respectively) have overlapping but unique tissue expression patterns. PHD2 is ubiquitously expressed, and whole-body disruption of PHD2 leads to early embryonic lethality. PHD1 and PHD3 are more selectively expressed, and mice with whole-body knockout of PHD1 or PHD3 are viable (3). The role of HIF signaling in the immune system has been a subject of intense research and debate, but the mechanisms have remained unclear.

\section{Oxygen-sensing pathways in immune cells}

Tissue hypoxia and HIF activation occur following tissue injury, infection, inflammation, and rapid tumor growth. Resident or recruited immune cells must rapidly activate HIF to adapt and function in a hypoxic environment. In tissues that demonstrate a basal hypoxic response (e.g., the intestine), physiological HIF expression is essential to maintain immune tolerance and function, which are critical for tissue homeostasis

\section{Related Article: p. 3640}

Conflict of interest: WZ has served as a consultant or advisor for Lycera, NGM Biopharmaceuticals, Synlogic, CStone Pharmaceuticals, and Henlix Biotech.

Copyright: @ 2019, American Society for Clinical Investigation.

Reference information: / Clin Invest. 2019;129(9):3524-3526. https://doi.org/10.1172/JCI130009.

(4). During a robust injury or in the setting of chronic inflammation, including cancer, pathological hypoxia via constitutive HIF expression leads to the activation of proinflammatory mediators and tissue damage. The role of hypoxia and HIF activation in immune cells is complex and may be celltype dependent. It is clear that hypoxia and HIF signaling affect metabolic pathways, in particular by inducing glycolytic gene expression, but the functional responses differ in different cell types. Therefore, many studies have utilized Cre-mediated deletion to understand the role of HIFs in individual immune cell populations, which have demonstrated the cell-autonomous dependency of HIFs in modulating immune cell function. However, those studies lacked temporal regulation, and because of lineage- and developmental stage-specific activation of various Cre recombinases, the results have at times been discordant (512). Furthermore, the approach of targeting HIF function in specific cell types does not address the role of heterocellular crosstalk in the response to hypoxia in the inflammatory or injured foci or in cancer.

In this issue, Yamamoto, Hester, and colleagues developed an inducible mouse model to assess the hypoxia/HIF pathway using an RNA interference knockdown approach in vivo (13). Specifically, they cloned reverse tetracycline transactivator $(r t T A)$ driven by the endogenous ROSA26 promoter and a single-copy tetracycline response element $(T R E)$ controlling a GFP-shRNA cassette expressing an shRNA sequence for PHD2, HIF1 $\alpha$, or HIF2 $\alpha$ downstream of the collagen type I (Colla1) gene. Upon doxycycline treatment, the mice expressed the shRNAs and GFP, enabling temporal and reversible gene silencing and cellular tracking in adult mice (Figure 1). The authors found that mice with knockdown of Phd2 became grossly sick, characterized by weight loss, alopecia, and anemia. The anemia was surprising, as HIF activation is known to increase erythropoi- 


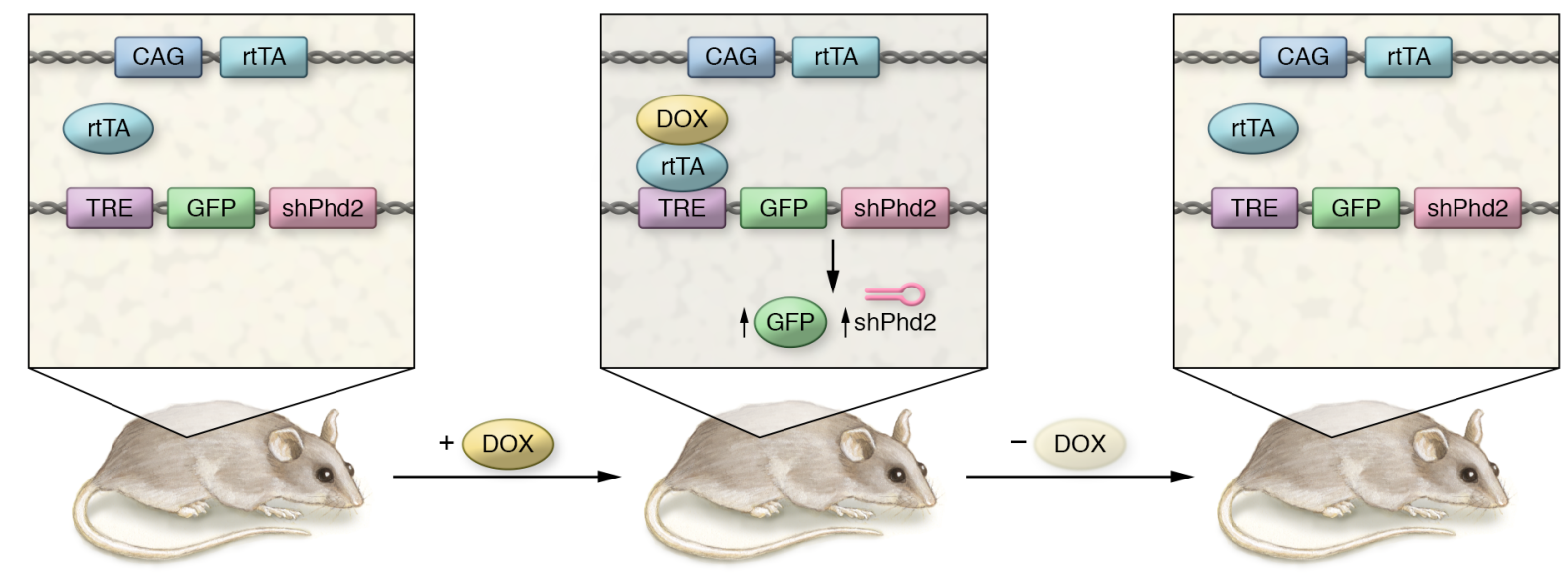

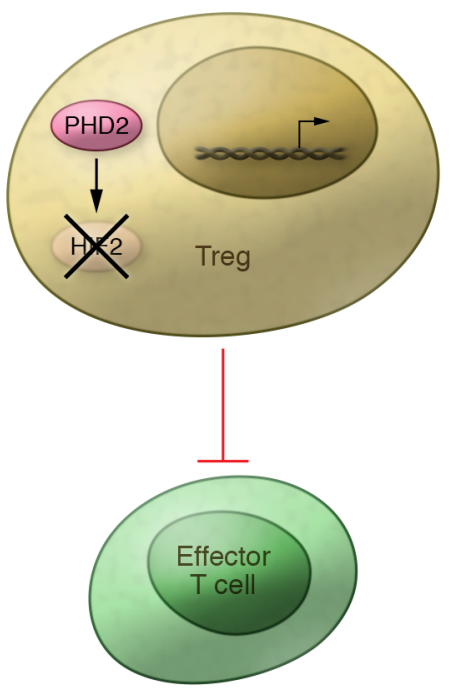

No autoimmunity
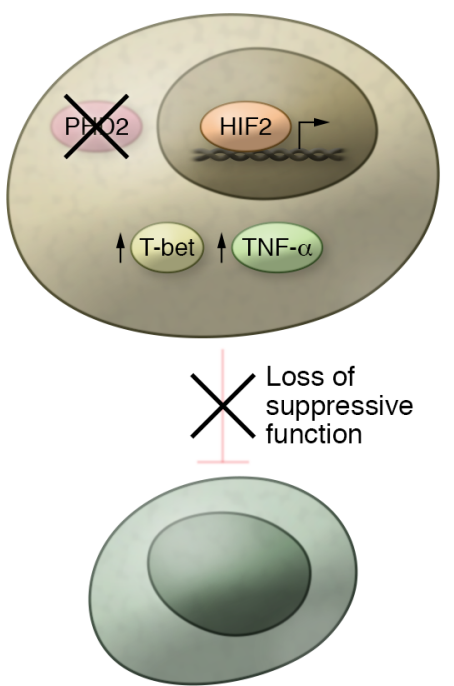

Leukocytosis Autoimmunity
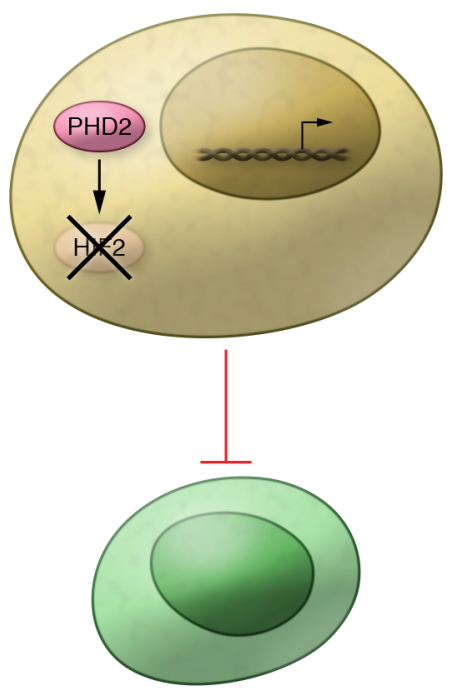

Reversal of autoimmunity

Figure 1. Transgenic loss of PHD2 results in autoimmunity. In the presence of doxycycline, transgenic mice show increased expression of shRNA for PHD2 in all tissues. shRNA for PHD2 (shPhd2) stabilizes HIF2 $\alpha$ in the hemopoietic compartment, leading to complete loss of the immunosuppressive function of Tregs. No changes in Foxp3 expression or Treg numbers, but an increase in T-bet and TNF- $\alpha$ expression, were observed following shPhd2 expression. The mice had a progressive autoimmune disorder, which was completely reversible following the removal of doxycycline (DOX). CAG, chicken $\beta$-actin.

esis by direct activation of erythropoietin, leading to increased iron absorption (14, 15). However, shPhd2 mice demonstrated lymphadenopathy, splenomegaly, and expansion of lymphoid compartments. Prominent immune infiltrates, inflammation, and injury were observed in a number of tissues. The findings were consistent in two transgenic lines with two different shRNAs targeting PHD2. It should be noted that this phenotype differs from reports of whole-body Cre-mediated inactivation of $P h d 2$ in adult mice, which led to death due to robust global changes in erythropoiesis and metabolism $(16,17)$. However, in a complementary approach, Yamamoto, Hester, and colleagues crossed tamoxifen-inducible Cre recombinase (Rosa26Cre ${ }^{E R T 2}$ ) mice with $P h d 2^{f / f l}$ mice and showed that four weeks following disruption of $P h d 2$, the mice exhibited peripheral lymphadenopathy and increased leukocytes, although the phenotype was less severe than that observed in the shPhd2 mice. The leukocytosis, lymphoid expansion, and immune dysregulation were reversed when doxycycline treatment was removed, indicating acute and reversible regulation of the response by PHD2. Likewise, simultaneous knockdown of $P h d 2$ and Hif $2 a$ rescued the effect of $P h d 2$ knockdown (whereas simultaneous knockdown of Phd2 and Hifla did not), demonstrating that the phenotype was at least partially dependent on HIF2 $\alpha$. The affinity and tissue specificity of the three PHDs for HIF1 $\alpha$ and HIF2 $\alpha$ in vivo are currently not known but could contribute to the selectivity of HIF activation following hypoxia.

\section{PHD2, Tregs, and autoimmunity}

The phenotype of the shPhd 2 mice was consistent with that of a progressive autoimmune disorder. To determine whether what they observed was immune cell autonomous, the authors generated bone marrow chimeras and showed that transfer of hemopoietic cells from shPhd 2 mice was sufficient to induce the immune phenotype in the recipients. Examination of $\mathrm{T}$ cell subsets in shPhd 2 mice revealed an increase in the absolute numbers of these cells, including a surprising increase in the number of Tregs, which maintain homeostasis and self-tolerance. Despite this finding, shPhd2 mice resembled other models with gross pathologies associated with disruption of Tregs. 
Further study by Yamamoto, Hester, and coworkers using a model of skin allograft rejection demonstrated that Tregs isolated from shPhd2 mice were unable to suppress $\mathrm{T}$ effector function, leading to allograft rejection and impaired survival (Figure 1). They complemented this work with in vitro assays that demonstrated a complete loss of Treg function, despite maintenance of Foxp3 expression. These data are in contrast to a study showing the effect of $V h l$ inactivation using a Foxp3-driven Cre, which resulted in reduced Foxp3 expression and an inflammatory phenotype, dependent on HIF1 $\alpha$ (18). This discrepancy may be related to differences between temporal knockdown compared with constitutive disruption via Cre-mediated deletion and to differences in PHD2/HIF2 $\alpha$ signaling compared with the HIF1 $\alpha$-mediated processes implicated in the $V h l$ inactivation model. Although the authors suggest a cell-autonomous role for PHD2 in Foxp $3^{+}$cells, future work crossing their line with a Foxp3-dependent system is needed to better understand the direct role of PHD2 in Tregs. In addition, given the autoimmune phenotype in shPhd 2 mice and the reported role of HIF in Th17 cells $(6,9,19)$, future studies should examine the Th17 cell compartment in shPhd 2 mice.

Inhibition of mitochondrial complex III leads to increased hypoxic metabolite 2-hydroxyglutarate (2-HG), which has also been shown to suppress Treg function. In that setting, expression of Foxp3 expression is not altered, but several immunoregulatory genes are inhibited (20). Although Yamamoto, Hester, and colleagues did not identify the mechanism by which downregulation of PHD2 suppresses Treg function, the changes in cytokine expression were consistent with those observed upon mitochondrial complex III inhibition. Immune responses require aerobic glycolysis to promote $\mathrm{T}$ cell proliferation and effector function, and immunometabolism and immune cell function are integrated via HIF signaling (21). Therefore, it would be interesting to determine whether the phenotype of the Tregs from shPhd2 mice is dependent on cellular metabolic pathway alteration.

\section{Therapeutic implications}

Currently, many orally active PHD inhibitors are in clinical trials involving patients with chronic kidney disease (CKD), for whom the inhibitors are intended to stimulate erythropoiesis to improve anemia. PHD inhibitors have so far been shown to be safe and effective in preclinical models and in patients (22). Recently, a battery of publications has shown that PHD inhibitors are beneficial in models of intestinal inflammation (23), and there is interest in assessing their action in patients with inflammatory bowel disease, which may require higher doses than those used to treat CKD. The work of Yamamoto, Hester, and coworkers argues for some caution and suggests that it will be important to monitor Treg function in patients treated with PHD inhibitors. Furthermore, since PHD inhibitors may affect Tregs, it would be interesting to test the antitumor potential of these drugs in syngenic models of cancer. In summary, the work highlights the need for further studies of the role of PHD2 and HIF $2 \alpha$ inhibitors in autoimmune disorders, inflammation, and cancer.

\section{Acknowledgments}

This work was supported by NIH grants CA148828 and DK095201 (to YMS) and CA217648, CA123088, CA099985, CA193136, and CA152470 (to WZ) and by the NIH through the University of Michigan Rogel Cancer Center grant CA46592 (to YMS and WZ).

Address correspondence to: Yatrik M. Shah, University of Michigan, Medical Science Building II, Ann Arbor, Michigan, 48108, USA. Phone: 734.615.0567. Email: shahy@umich.edu. Or to: Weiping Zou, University of Michigan, Biomedical Science Research Building, Ann Arbor Michigan, USA. Phone: 734.615.5554, Email: wzou@med.umich.edu.

\footnotetext{
1. Prabhakar NR, Semenza GL. Oxygen sensing and homeostasis. Physiology (Bethesda). 2015;30(5):340-348.

2. Kaelin WG. Proline hydroxylation and gene expression. Annu Rev Biochem. 2005;74:115-128.

3. Ivan M, Kaelin WG. The EGLN-HIF O. Mol Cell. 2017;66(6):772-779.

4. Colgan SP, Taylor CT. Hypoxia: an alarm signal during intestinal inflammation. Nat Rev Gastroenterol Hepatol. 2010;7(5):281-287.

5. Clambey ET, et al. Hypoxia-inducible factor-1 alpha-dependent induction of FoxP3 drives regulatory $\mathrm{T}$-cell abundance and function during inflammatory hypoxia of the mucosa. Proc Natl Acad Sci U S A. 2012;109(41):E2784-E2793.

6. Dang EV, et al. Control of T(H)17/T(reg)
}

balance by hypoxia-inducible factor 1. Cell. 2011;146(5):772-784.

7. Doedens AL, et al. Hypoxia-inducible factors enhance the effector responses of CD8(+) T cells to persistent antigen. Nat Immunol. 2013;14(11):1173-1182.

8. Lukashev D, et al. Cutting edge: hypoxia-inducible factor 1alpha and its activation-inducible short isoform I.1 negatively regulate functions of $\mathrm{CD}^{+}$and $\mathrm{CD}^{+} \mathrm{T}$ lymphocytes. J Immunol. 2006;177(8):4962-4965.

9. Shi LZ, et al. HIF1alpha-dependent glycolytic pathway orchestrates a metabolic checkpoint for the differentiation of TH17 and Treg cells. J Exp Med. 2011;208(7):1367-1376.

10. Takeda N, et al. Differential activation and antagonistic function of HIF-\{alpha\} isoforms in macrophages are essential for NO homeostasis. Genes Dev. 2010;24(5):491-501.

11. Thompson AA, et al. Hypoxia determines survival outcomes of bacterial infection through HIF-1alpha dependent re-programming of leukocyte metabolism. Sci Immunol. 2017;2(8):eaal2861.

12. Wang H, Flach H, Onizawa M, Wei L, McManus MT, Weiss A. Negative regulation of Hifla expression and TH17 differentiation by the hypoxia-regulated microRNA miR-210. Nat Immunol. 2014;15(4):393-401.

13. Yamamoto A, et al. Systemic silencing of $P h d 2$ causes reversible immune regulatory dysfunction. JClin Invest. 2019;129(9):3640-3656.

14. Wang GL, Semenza GL. Characterization of hypoxia-inducible factor 1 and regulation of DNA binding activity by hypoxia. J Biol Chem . 1993;268(29):21513-21518.

15. Schwartz AJ, et al. Hepatic hepcidin/intestinal HIF- $2 \alpha$ axis maintains iron absorption during iron deficiency and overload. J Clin Invest. 2019;129(1):336-348.

16. Takeda K, et al. Regulation of adult erythropoiesis by prolyl hydroxylase domain proteins. Blood. 2008;111(6):3229-3235.

17. Takeda K, Cowan A, Fong GH. Essential role for prolyl hydroxylase domain protein 2 in oxygen homeostasis of the adult vascular system. Circulation. 2007;116(7):774-781.

18. Lee JH, Elly C, Park Y, Liu YC. E3 Ubiquitin ligase VHL regulates hypoxia-inducible factor- $1 \alpha$ to maintain regulatory $\mathrm{T}$ cell stability and suppressive capacity. Immunity. 2015;42(6):1062-1074.

19. Kryczek I, et al. Human TH17 cells are longlived effector memory cells. Sci Transl Med. 2011;3(104):104ra100.

20. Weinberg SE, et al. Mitochondrial complex III is essential for suppressive function of regulatory $\mathrm{T}$ cells. Nature. 2019;565(7740):495-499.

21. Halligan DN, Murphy SJ, Taylor CT. The hypoxiainducible factor (HIF) couples immunity with metabolism. Semin Immunol. 2016;28(5):469-477.

22. Sakashita M, Tanaka T, Nangaku M. Hypoxiainducible factor-prolyl hydroxylase domain inhibitors to treat anemia in chronic kidney disease. Contrib Nephrol. 2019;198:112-123.

23. Colgan SP, Campbell EL, Kominsky DJ. Hypoxia and mucosal inflammation. Annu Rev Pathol. 2016;11:77-100. 